\title{
ACRL Nominating Committees
}

The ACRL Committee on Appointments and Nominations and the ACRL section nominating committees are seeking candidates for nomination for election to office and for recommendation for appointment to committees. Persons interested in making recommendations or in being considered for nomination or appointment should contact a member of the appropriate committee before the Midwinter Meeting.

\section{ACRL}

Jane G. Flener, Associate University Librarian, University of California, Berkeley, CA 94720, Chairman

Robert K. Bruce, Librarian, Carleton College, Northfield, MN 55057

Florence S. DaLuiso, Art Librarian, Harriman Library, State University of New York at Buffalo, Buffalo, NY 14214

Rosemary N. Henderson, Director of Library Services, Coffeyville Community Junior College, Coffeyville, KS 67337

Adrian Jones, Director of Libraries, Roosevelt University, 430 S. Michigan Ave,, Chicago, IL 60605

Charles M. Schaeve, Life Sciences Librarian, Purdue University, Lafayette, IN 47907

Clarence R. Walters, County Librarian, Contra Costa County Library System, 1750 Oak Park Blvd., Pleasant Hill, CA 94523

Five to be appointed.

\section{AGRICULTURE AND BIOLOGICAL SCIENCES SECTION}

Charles M. Schaeve, Life Sciences Librarian, Purdue University, Lafayette, IN 47907, Chairman

Joan T. Hicks, Librarian, Veterinary Medicine Library, University of Illinois, Urbana, IL 61801

Donna K. Rudolph, Route 1, Box 341, Blacksburg, VA 24060

\section{ANTHROPOLOGY SECTION}

To be appointed.

\section{ART SECTION}

Florence S. DaLuiso, Art Librarian, Harriman Library, State University of New York at Buffalo, Buffalo, NY 14214, Chairman

Mary Ashe, Art and Music Librarian, San

Francisco Public Library, Civic Center, San Francisco, CA 94102

Stephen G. Matyi, Head, Fine Arts Department, Cleveland Public Library, 325 Superior Ave., Cleveland, $\mathrm{OH} 44114$

\section{ASIAN AND AFRICAN SECTION}

Adrian Jones, Director of Libraries, Roosevelt University, $430 \mathrm{~S}$. Michigan Ave., Chicago, IL 60605, Chairman

Lee S. Dutton, Southeast Asia Librarian, Swen Franklin Parson Library, Northern Illinois University, DeKalb, IL 60115

T. H. Tsien, Curator, Far Eastern Library, University of Chicago, Chicago, IL 60637

\section{COLLEGE LIBRARIES SECTION}

Robert K. Bruce, Librarian, Carleton College, Northfield, MN 55057, Chairman

Barbara G. LaMont, Librarian, Vassar College, Poughkeepsie, NY 12601

Sr. M. Franz Lang, Director of Library Service, Barry College, Miami, FL 33161

\section{COMMUNITY AND JUNIOR COLLEGE LIBRARIES SECTION}

Rosemary N. Henderson, Director of Library Services, Coffeyville Community Junior College, Coffeyville, KS 67337, Chairman

Charlotte Brennan, Director of Library Services, Yavapai College, 1100 E. Sheldon, Prescott, AZ 86301

Gloria H. Terwilliger, Director, Learning Resources, Northern Virginia Community College, Alexander Campus, 3001 N. Beauregard St., Alexandria, VA 22311

\section{EDUCATION AND BEHAVIORAL SCIENCES SECTION}

To be appointed.

\section{LAW AND POLITICAL SCIENCE SECTION}

Clarence R. Walters, County Librarian, Contra Costa County Library System, 1750 Oak Park Blvd., Pleasant Hill, CA 94523, Chairman

Harold H. Erickson, Director of the Library, University of Nevada, Las Vegas, 4505 Maryland Parkway, Las Vegas, NV 89109

M. Sharon McClure, Government Documents Librarian, University of California, San Diego, La Jolla, CA 92037

\section{RARE BOOKS AND MANUSCRIPTS SECTION}

To be appointed.

\section{SLAVIC AND EAST EUROPEAN SECTION}

To be appointed.

\section{UNIVERSITY LIBRARIES SECTION}

To be appointed. 